\title{
Tips and Techniques: Conical Shaping of Structural Allografts for Bone Block Arthrodesis in Failed First Metatarsophalangeal Joint Arthroplasty
}

\author{
by Ronald Belczyk, DPM ${ }^{1} \rrbracket$, Damon Combs, DPM ${ }^{2}$, Dane K. Wukich, MD $^{3} \square$
}

The Foot \& Ankle Journal 1 (8): 4

The purpose of this article is to report on a technical tip when performing bone block arthrodesis following failed first metatarsophalangeal arthroplasty. Conical reaming of structural allografts permits proper toe positioning, is reproducible, and has a high rate of fusion.

Key words: Bone block arthrodesis, foot surgery revision, first MPJ fusion

Accepted: July 2008

Published: August 2008

This is an Open Access article distributed under the terms of the Creative Commons Attribution License. It permits unrestricted use, distribution, and reproduction in any medium, provided the original work is properly cited. @The Foot \& Ankle Journal (www.faoj.org)

The use of structural autograft is commonly used in revision first metatarsophalangeal fusion. ${ }^{1}$ This article reports a case of a failed metatarsophalangeal arthroplasty and provides a technique by which structural allograft can be prepared with conical reamers to fill a substantial osseous defect created during revision surgical intervention. Radiographs of a healthy 60 year old patient suffering from a painful total joint arthroplasty are shown in Figures 1.

Address correspondence to: Dane Wukich, MD. UPMC

Comprehensive Foot and Ankle Center. Roesch-Taylor Bldg Ste 7300.

2100 Jane St. Pittsburgh, PA 15203. Phone: 412-586-1546 Fax: 412-

586-1544

Email:wukichdk@upmc.edu

\footnotetext{
${ }^{1}$ PGY-4, Fellow, Foot and Ankle Surgery, University of Pittsburgh Medical Center, Pittsburgh, Pennsylvania, 15203.

${ }^{2}$ Resident, Foot and Ankle Surgery, University of Pittsburgh Medical Center, Pittsburgh, Pennsylvania, 15203.

${ }^{3}$ Chief, Foot and Ankle Division, University of Pittsburgh Medical Center Department of Orthopedic Surgery and Assistant Professor, University of Pittsburgh School of Medicine, Pittsburgh, Pennsylvania, 15203.
}

During revisional surgery of the first metatarsophalangeal joint, a defect is created from both the removal of the implant and preparation of the osseous surfaces. Preoperatively, the void can be estimated by measuring the distance from the proximal and distal bone segments.

The anticipated defect can be filled with an interpositional bone graft. Some considerations with this type of surgery include not only restoring length but also maintaining alignment and providing structural stability. Preparation of an interpositional bone graft is often fashioned in a rectangular or peg- in- hole fashion, but these techniques may limit proper alignment or surface contact area between the bone graft and adjacent bony fragments. Conical reaming of the graft allows for more degrees of motion for toe positioning, is reproducible, and has a higher rate of fusion. ${ }^{2,3}$ Traditionally, non-contained defects that require structural graft have been filled with corticocancellous autograft harvested from the iliac crest. 


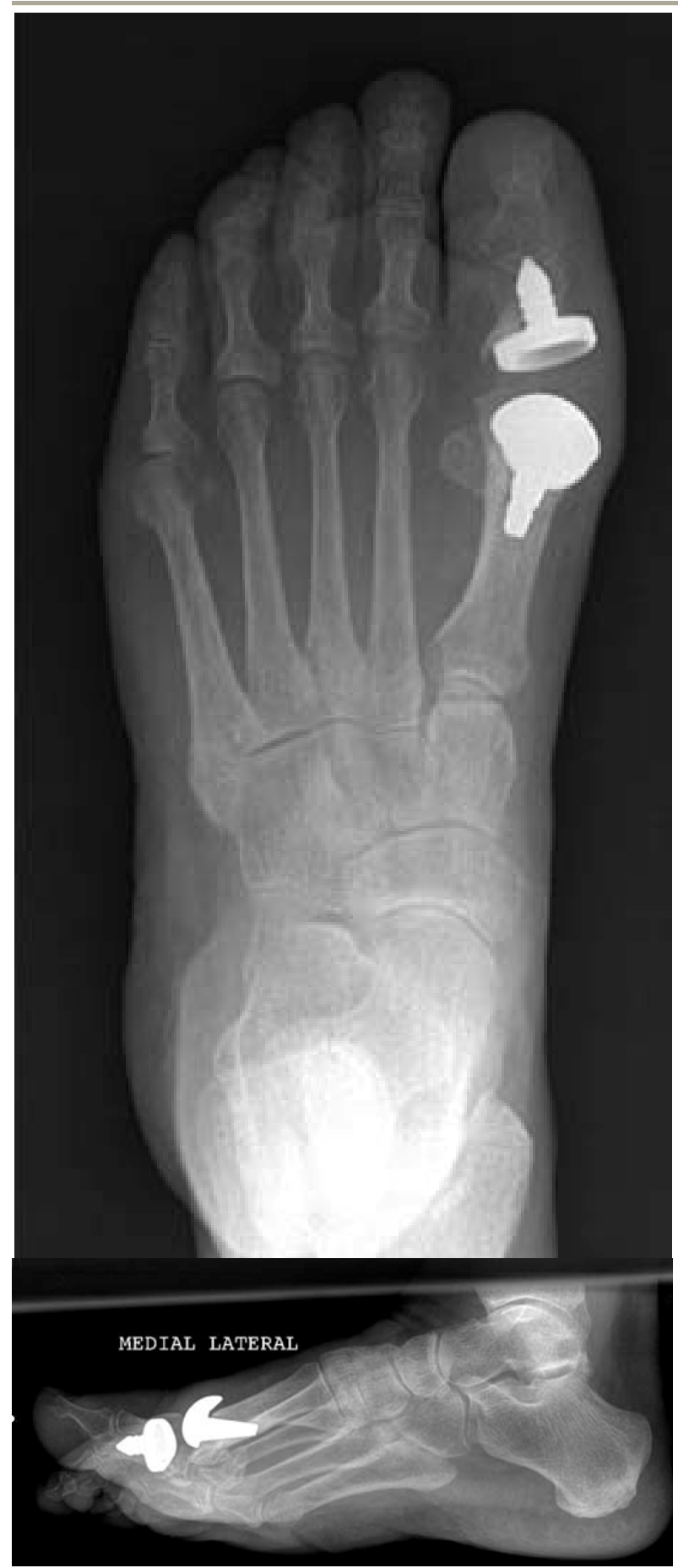

Figures 1 AP and Lateral radiographs of the left foot demonstrating first metatarsophalangeal arthroplasty. The stem of the phalanx prosthesis is partially outside the posterior cortex of the phalanx. The implant was removed to due to pain and instability from the implant.

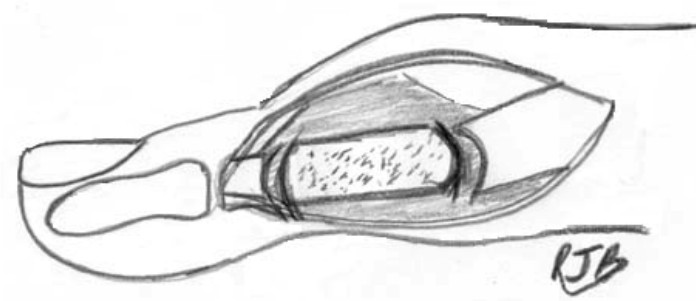

Figure 2 Conical reaming of the structural bone.

However, there is significant morbidity at the donor site associated with this procedure such as bleeding, infection, nerve injury, and fracture. The use of structural allograft is helpful because there is an unlimited supply, has prolonged storage capacity, and is available in many sizes and shapes. Structural allograft can be obtained from cadaveric iliac crest, femoral head, or fibula. The use of allograft has been documented in tumor surgery and long term studies show no statistically significant difference in the morphology of repair between autograft and allograft. ${ }^{4,5}$

\section{Surgical Technique}

Full thickness skin flaps are created over the first metatarsophalangeal joint and the implant is then removed. Proper joint debridement is obtained by removing any fibrous tissue and resecting the proximal and distal bony segments down to bleeding bone edges with the reamer. This is frequently referred to as the paprika sign. If deep infection is suspected but no obvious purulence is visible, soft tissue can be sent for a frozen section. In the absence of neutrophils under high powered field, definitive surgery can be performed safely. The proximal and distal bony segments are resurfaced in the shape of a cup. Figure 2 illustrates how this is performed. 


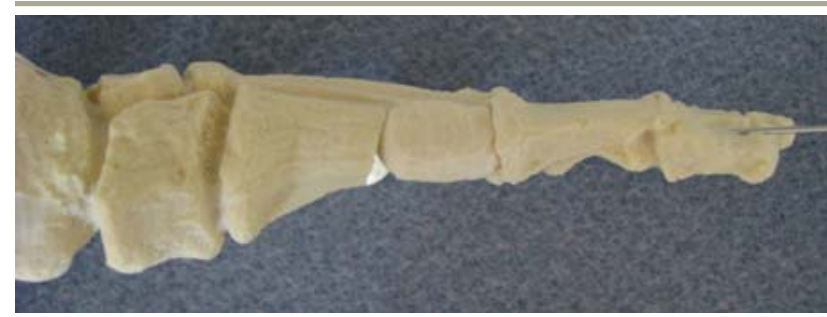

Figure 3 Lateral view of a conically reamed structural bone graft on a corticocancellous saw bone model.

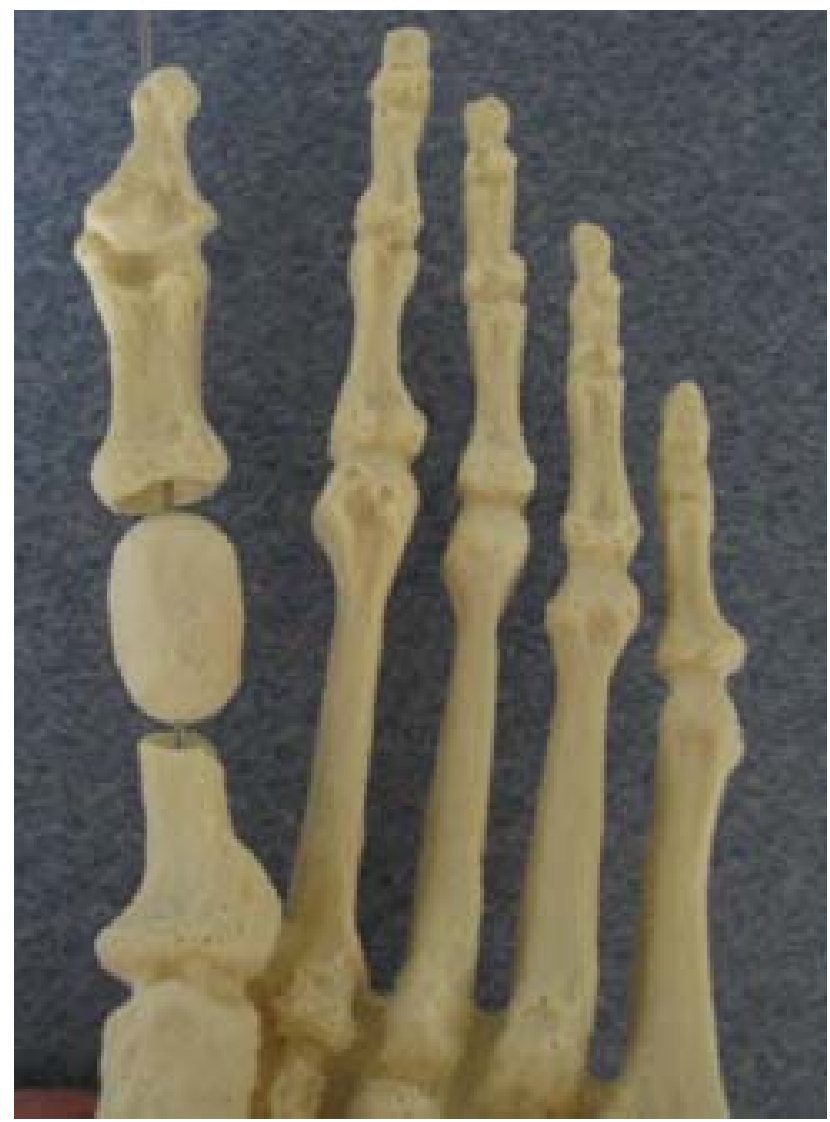

Figure 4 Plantar view of a conically reamed structural bone graft on a corticocancellous saw bone model.

A structural allograft of appropriate length can then be obtained. The case presented in this article utilized cadaveric iliac crest. This graft is then fashioned using cup and cone reamers. The graft can then be inserted and the toe properly aligned in the saggittal, frontal and transverse planes. Figures 3 and 4 show how this graft is properly fit and temporary fixated on a corticocancellous saw bone model.

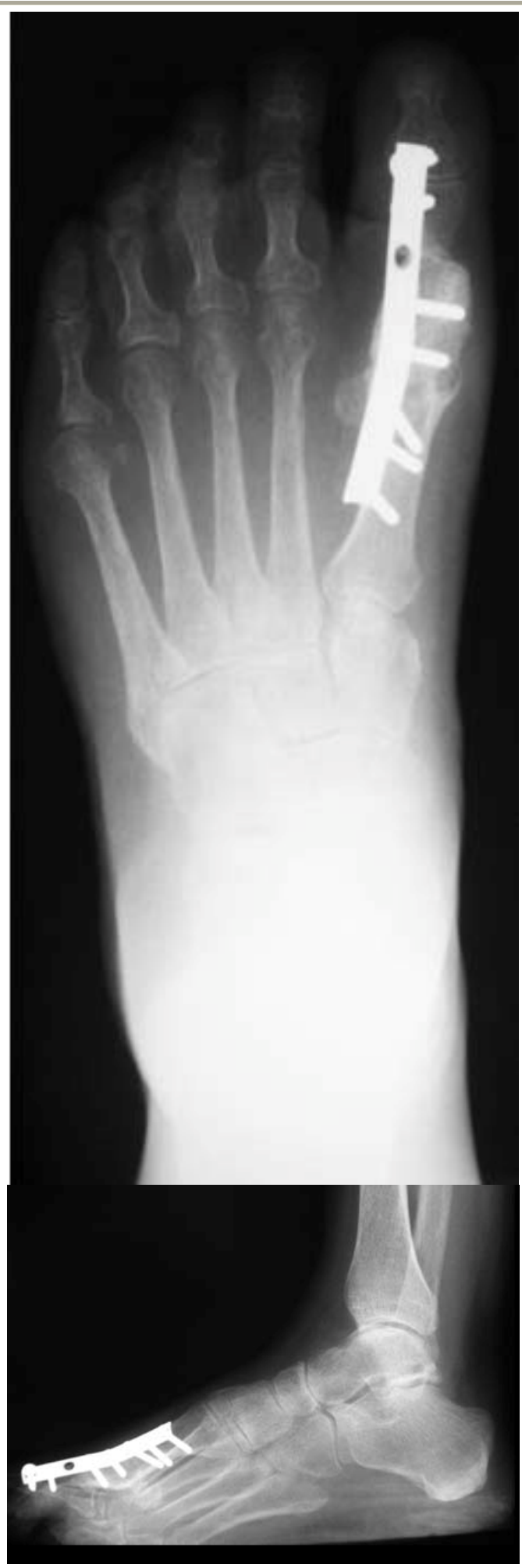

Figures 5 AP and lateral radiographs of a left foot one year following first metatarsophalangeal fusion using iliac crest allograft that was fashioned with a cup and cone configuration. 


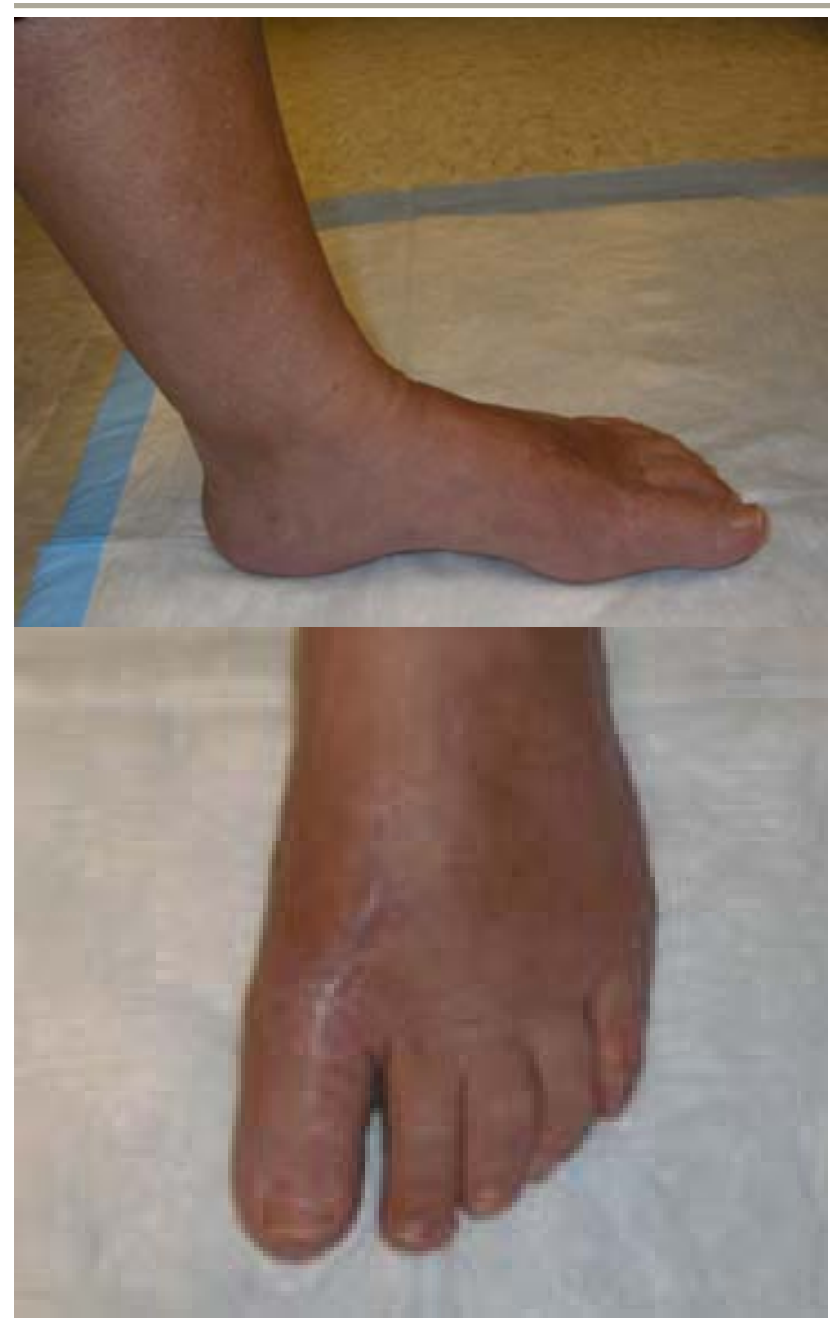

Figures 6 Clinical pictures one year following first metatarsophalangeal fusion with interpositional bone graft with iliac crest allograft.

Fixation can then be obtained using a $1 / 3$ tubular plate or locking plate system, preferable placed on the dorsal surface. Postoperatively, the limb is kept unloaded for eight weeks and then partial weight bearing with cast immobilization until radiographic consolidation is present at both graft interfaces. Figures 5 and 6 demonstrate clinical and radiographic appearance of our patient at one year follow-up. In summary, this clinical tip can be useful for the foot and ankle surgeon specializing in revisional surgery.
This technique limits morbidity at the donor site and has been used successfully in restoring length, maintaining alignment, and providing structural stability of defects created with this type of surgery.

\section{References}

1. Neufeld S, Uribe J, Myerson M., Use of structural allograft to compensate for bone loss in arthrodesis of the foot and ankle Foot and Ankle Clinics, 7: p. 1-17, 2002.

2. Herr M, Kile T., First Metatarsophalangeal Joint Arthrodesis with Conical Reaming and Crossed Dual Compression with Screw Fixations Techniques in Foot and Ankle Surgery, 4(2): p. 8594, 2005.

3. Yu G, Gorby P., First metatarsophalangeal joint arthrodesis Clin Podiatr Med Surg, 21:p.65-96, 2004.

4. Goldberg VM, Stevenson S., Natural history of autografts and allografts. Clin Orthop, 225:p.7-16, 1987.

5. Reynolds FC, Oliver DR., Experimental evaluation of homogenous bone grafts. J Bone Joint Surg Am, 32:p.283-297, 1950. 